\title{
TOPOLOGICAL OPTIMIZATION OF UNDER-PLATFORM DAMPERS WITH MOVING MORPHABLE COMPONENTS AND GLOBAL OPTIMIZATION ALGORITHM FOR NONLINEAR FREQUENCY RESPONSE
}

\author{
E. Denimal \\ Department of Mechanical Engineering \\ Imperial College London \\ London SW7 2AZ, UK \\ Email: e.denimal@imperial.ac.uk \\ C. Wong \\ Rolls-Royce plc \\ Derby DE2 48J, UK \\ Email: Chian.Wong@Rolls-Royce.com
}

\author{
F. El Haddad \\ Department of Mechanical Engineering \\ Imperial College London \\ London SW7 2AZ, UK \\ Email: f.el-haddad@imperial.ac.uk
L. Salles
Department of Mechanical Engineering
Imperial College London
London SW7 2AZ, UK \\ Email: I.salles@imperial.ac.uk
}

\section{ABSTRACT}

To limit the risk of High Cycle Fatigue, underplatform dampers are traditionally used in aircraft engines to control the level of vibration. Many studies demonstrate the impact of the geometry of the damper on its efficiency, thus the consideration of topological optimization to find the best layout of the damper seems natural. Because of the nonlinear behaviour of the structure due to the friction contact interface, classical methods of topological optimization are not usable. The present study proposes to optimize the layout of an underplatform damper to reduce the level of nonlinear vibrations computed with the MultiHarmonic Balance Method. The approach of topological optimization employed is based on the Moving Morphable Components framework together with the Kriging and the Efficient Global Optimization algorithm to solve the optimization problem. The results show that the level of vibration of the structure can be reduced of $30 \%$ and allow for the identification of different efficient geometries.

$\begin{array}{cl}\text { ACRONYMS } & \\ \text { AFT } & \text { Alternate Frequency/Time } \\ \text { DoE } & \text { Design of Experiments } \\ \text { DOF } & \text { Degree Of Freedom } \\ \text { EGO } & \text { Efficient Global Optimisation } \\ \text { FRF } & \text { Frequency Response Function } \\ \text { HCF } & \text { High Cycle Fatigue } \\ \text { HPT } & \text { High Pressure Turbine } \\ \text { LHS } & \text { Latin Hypercube Sampling } \\ \text { LSF } & \text { Level-Set Function } \\ \text { LSM } & \text { Level-Set Method } \\ \text { MHBM } & \text { Multi-Harmonic Balance Method } \\ \text { MMC } & \text { Moving Morphable Components } \\ \text { TO } & \text { Topological Optimisation } \\ \text { UPD } & \text { UnderPlatform Damper }\end{array}$

\section{INTRODUCTION}

In the aerospace industry, the requirements for the design of engines are becoming more stringent and new engines must be more efficient and lighter. Thus, each component tends to reach its structural limits. Considering the turbine blades, they are submitted to high level of exposure to thermal stresses, centrifugal stresses or vibrational stresses [1,2]. The latter can lead to High Cycle Fatigue (HCF) and can potentially lead to a failure of the 
blades [3]. Because of the large operating speed range and the high modal density of bladed disks, all the critical resonances cannot be avoided. For this reason, it is crucial to limit the level of vibrations at resonance. The most widely used solution are based on friction damping [4-6].

Dry friction can be introduced in different locations of the structure (shrouds, roots, blade tips), but the most efficient solution relies on the use of Underplatform Dampers (UPD) [6, 7]. They are located in the groove under the platforms of two adjacent blades and consist in a small metal device. They remain in contact with the platforms due to the centrifugal loading. When the blades vibrate, the relative displacement between the platforms and the UPD generates friction and so energy is dissipated $[8,9]$.

The geometry of the UPD has a significant impact on its effectiveness. Indeed, many studies are related to the study of the comparison of different geometries. The most classical geometries are based on a wedge geometry [10-12], a cylindrical geometry $[11,13]$ or a mix of them [14]. From those studies, it appears that the shape and the geometry of the damper has a high impact on its efficiency.

Topological Optimization (TO) for continuum structures consists in the identification of a sub-domain occupied by the structure in a total available domain where only the boundary conditions are known $[15,16]$. The topology of a component is defined by its internal and external boundaries and by the number of inner holes. These characteristics are optimized simultaneously with respect to a predefined objective and constraint functions. TO is mostly used in a pre-design stage to identify the most efficient layout of the structure. The two most classical methods of TO are the density-based approach and the Level Set Method (LSM). The first method consists in the optimization of the element densities of a mesh describing the design space [17, 18]. The second one is based on an implicit description of the material boundaries with a Level Set Function (LSF), which is often propagated by solving a Hamilton-Jacobi equation [19, 20]. These two approaches require the sensitivities, of the densities or of the shape, with respect to the objective function and the constraints. These sensitivities might be impossible to determine analytically for some problems and are too expensive to be determined numerically.

A recent approach, called the Moving Morphable Components (MMC) framework, was proposed by Guo et al. [21]. The basic idea relies on the fact that the topology can be described by the association of elementary beams. Hence, the material domain is still described by an LSF which is decomposed in local LSFs that describe each beam. The expression of these local LSF is explicit and depends on a few parameters. Hence, the size of the optimization problem is drastically reduced, and it gives the opportunity to consider global optimisation methods. Thus, in 2019, Raponi et al. [22], proposed to use the Kriging meta-modelling method together with the Efficient Global Opti- mization (EGO) algorithm [23] to solve a problem of topological optimisation for crash-worthiness where the sensitivities cannot be determined.

Considering vibration issues, TO has been applied to the optimisation of eigenvalues in a linear context in [24-27] with the different methods presented previously. The extension to the non-linear case is done in [28,29] where the width of a clamped-clamped beam is optimised (constant thickness) and geometric non-linearities are considered. Gradient-based optimisation is used for the optimisation. Considering contact problems, the problem of TO has been addressed in a quasi-static context in [30-32]. The objective of this study is to perform TO for the optimisation of nonlinear dynamic systems in the presence of a friction interface, which has never been done before according to the knowledge of the authors. Here, TO is applied on a UPD to identify the damper's layout that reduces to the maximum the levels of the nonlinear vibrations of the blades on a given frequency range. The optimization process must take into consideration the friction contact interface between the damper and the structure, but also the fact that the load applied on the UPD depends on its mass, and so on its geometry. Furthermore, the objective function of the problem is expected to be strongly non-convex with many local minima. For this reason, global optimisation is considered and the MMC framework with Krigingbased optimization is used to solve the current problem.

The objective of this paper is to investigate the capacity of the MMC framework coupled with global optimisation algorithm to optimise the topology of an UPD in order to reduce the amplitude of the nonlinear vibrations of the blades. Hence, the methodology is applied on a 2D system that simulates the behaviour of a real HPT blade as a preliminary work to examine the potential of the approach. The paper is organized as follows. First the optimisation problem is introduced. The mechanical system, the problem geometry and the methodology used to determine the nonlinear vibrations of the structure are presented. Secondly, the MMC framework and the optimization process based on Kriging and on the EGO algorithm are presented. Finally, the numerical results are presented.

\section{PROBLEM PRESENTATION}

The objective of this section is to present the physical problem that will be optimized. Hence, the mechanical system as well as the modelling choices and the numerical strategy for the prediction of the nonlinear vibrations are presented.

\section{Model Presentation}

The model used is based on the geometry of an underplatform test rig used and presented in [12]. It was designed to simulate the dynamic behaviour of the dynamic response of a real HPT blade and to study the impact of UPD on a blade-like struc- 


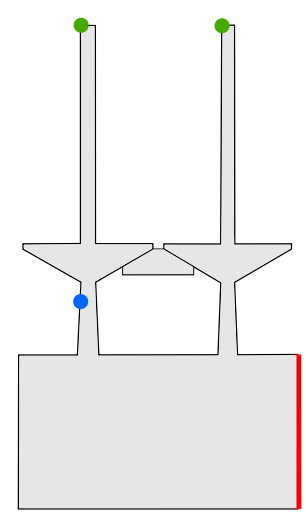

FIGURE 1: 2D geometry of the model - $(-)$ clamped - $(\bullet)$ excitation point - (•) output points

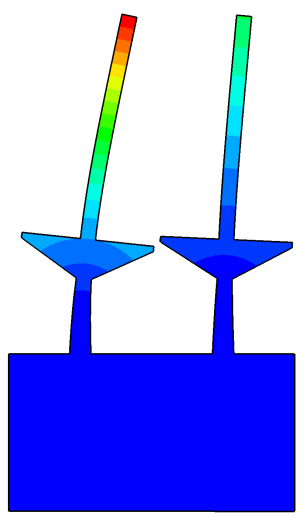

(a)

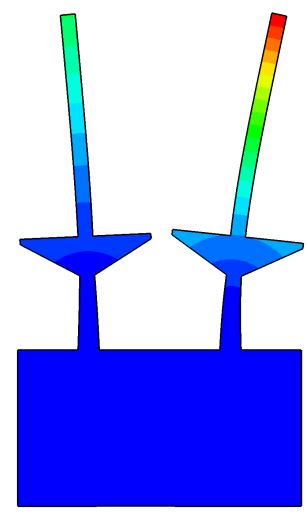

(b)
FIGURE 2: First two modes of the structure without the damper - (a) in-phase mode : $f_{1}=246.73 \mathrm{~Hz}$ and (b) out-of-phase mode: $f_{2}=247.51 \mathrm{~Hz}$

ture. The model considered here is based on the 2D geometry of this structure and is represented Figure 1. It is composed of two pseudo-beams fixed on a common base which simulates a rigid disk. To be consistent with the work done before in [12], the boundary conditions remain the same and the right side of the base is clamped for later experimental validations. A 2D geometry may seems simplistic, but the main concern of this study is to investigate the interest and the possibilities of using krigingbased optimisation for TO of nonlinear vibrations with friction. This simple case allows for much lower simulation times and a lower number of optimisation parameters.

The mesh of the blades is composed of 3324 8-node biquadratic plane strain elements. The structure is made of steel with a Young modulus of $197 \mathrm{GPa}$ and a density equal to $7800 \mathrm{~kg} / \mathrm{m}^{3}$. The study focuses on the first bending mode of the blades. Since there are two blades, their motion can be in- phase or out-of-phase. These two modes are represented in Figure 2 and the natural frequencies are equal to $246.73 \mathrm{~Hz}$ and $247.51 \mathrm{~Hz}$, respectively. It is worth noticing they are determined without the damper.

As a reference case, a wedge damper is located between the two platforms of the blades as represented in Figure 1. The centrifugal loading keeps it in place during operation. The mesh of the damper is represented in Figure 3 and is composed of 36048 node biquadratic plane strain elements. The mesh is constructed so that the contact points between the damper and the blades are matching. The number of elements may seem huge for this component, but this mesh will be used later in the process of the topological optimization and corresponds to the discretisation of the problem.

A node-to-node contact formulation is used in the problem and 51 contact points are present on each side of the damper. The dynamic behaviour at each contact node is modelled with a 1D friction contact element. It consists of one Jenkins element and one other spring to allow normal load variations. Each element is characterized by four parameters, namely the friction coefficient $\mu$, the tangential stiffness $k_{t}$, the normal stiffness $k_{n}$ and the initial pre-load $N_{0}$. The normal pre-load and the stiffness are normalized by unit of area, so each contact element has its own characteristics. During the cycle, the normal loading is kept constant and no separation is possible.

The contact pressure due to the centrifugal loading $C_{F}$ is modelled by a constant value [10] and the initial pressure $\sigma_{0}$ on each side of the damper is given by:

$$
\sigma_{0}=\frac{1}{2} \frac{C_{F}}{A(\cos \alpha+\mu \sin \alpha)}
$$

where $A$ is the contact area on each side, $\mu$ is the friction coefficient and $\alpha$ is the damper angle. The load $C_{F}$, aimed to simulate the centrifugal loading, is given by $C_{F}=m_{\text {damper }} \times R \times \omega^{2}$ where $m_{\text {damper }}$ is the damper mass, $R$ the radius and $\omega$ the rotational speed.

\section{Multi-Harmonic Balance Method}

The friction interface brings nonlinearity in the system and specific numerical strategies must be employed to determine the vibration levels. Thus, the method of the multi-harmonics balance (MHBM) coupled with a model reduction technique in-

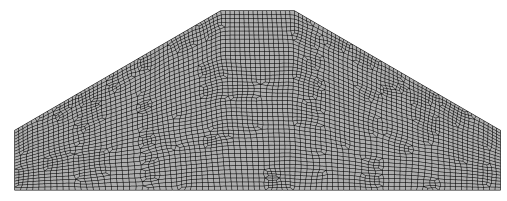

FIGURE 3: Mesh of the full damper 
cluded in the in-house code FORSE (FORce Response SuitE) is used in the present study. The latter has been presented in details in $[33,34]$. The reduction of the model is based on a FRF matrix representation of the model. This FRF matrix is determined by calculating its exact value at some frequency points and adding a second term that describes its variation in a range of frequencies [34].

The dynamic equation of the system can be written:

$$
\mathbf{M u ̈}+\mathbf{C} \dot{\mathbf{u}}+\mathbf{K u}+\mathbf{f}_{n l}(\mathbf{u}, \dot{\mathbf{u}})=\mathbf{p}(t)
$$

where $\mathbf{M}, \mathbf{C}$ and $\mathbf{K}$ are the mass, damping and stiffness matrices respectively. $\mathbf{f}_{n l}$ corresponds to the nonlinear forces vector due to the friction contact, $\mathbf{p}$ is the external forces vector and $\mathbf{u}$ is the displacements vector. The dot represents the derivative with respect to the time. As the external forces are periodic, it is natural to search for a periodic response for (2). Hence, the idea of the MHBM, is to write the problem on a Fourier series composed of $N_{h}$ retained harmonics. So, the response can be written:

$$
\mathbf{q}(t)=\mathbf{q}_{0}+\sum_{i=1}^{N_{h}} \mathbf{q}_{i}^{C} \cos \left(k_{i} \omega t\right)+\mathbf{q}_{i}^{S} \sin \left(k_{i} \omega t\right)
$$

where $\mathbf{q}_{0}$ is the constant term in the Fourier series, $\mathbf{q}_{i}^{C}$ are the cosine coefficients, $\mathbf{q}_{i}^{S}$ the sine coefficients and $k_{i}$ the $i^{t h}$ retained harmonic. The harmonics retained are chosen a priori by the user. By injecting the latter in Equation 2, and using the orthogonality of the Fourier series, the problem can be approximated by:

$$
\mathbf{Z}(\omega) \tilde{\mathbf{q}}+\tilde{\mathbf{f}}_{n l}=\tilde{\mathbf{p}}
$$

where $\mathbf{Z}$ is the dynamic stiffness matrix, $\tilde{\mathbf{q}}=$ $\left\{\mathbf{q}_{0}, \mathbf{q}_{1}^{C}, \mathbf{q}_{1}^{S}, \ldots, \mathbf{q}_{n}^{C}, \mathbf{q}_{n}^{S}\right\}$ is the vector of the Fourier coefficients, $\tilde{\mathbf{f}}_{n l}$ and $\tilde{\mathbf{p}}$ the nonlinear and the external efforts expressed in the Fourier basis, respectively. An AFT algorithm is used for the determination of the nonlinear forces Fourier coefficients [35]. The problem (4) is solved by using a Newton-Raphson algorithm. To find a continuous response curve with regard to the pulsation of excitation $\omega$, continuation is used. Here a secant predictor together with an arc-length corrector is used. In the following of the paper, harmonics 1 and 3 are retained for the MHBM simulations. This choice is based on a convergence study, not presented here for the sake of consistency.

\section{Application on the Initial Test Case}

The method of the MHBM is then used to compute the nonlinear dynamic response of the structure. The system is excited

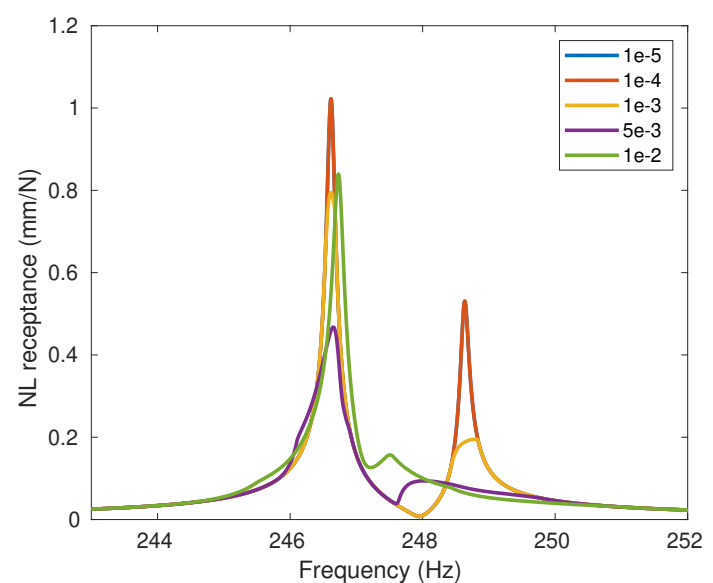

FIGURE 4: Nonlinear receptance at the point on the left blade for different excitation levels for a full damper

at the bottom of the left blade (blue point in Figure 1) and the displacements at the blades tips are determined (green points in Figure 1). A friction coefficient equal to 0.5 is chosen and the contact stiffness are taken equal to $k_{t}=k_{n}=0.5 \mathrm{~N} / \mathrm{mm}^{3}$. Different excitation levels are considered, from $1 e^{-5}$ to $1 e^{-2} \mathrm{~N}$ and the nonlinear receptances of the point at the tip of the left blade are given in Figure 4. For the full damper, the centrifugal loading $C_{F}$ is equal to $9.54 e^{-4} \mathrm{~N}$.

For an excitation force equals to $1 e^{-5} \mathrm{~N}$ or $1 e^{-4} \mathrm{~N}$, two resonant frequencies appear at $246.6 \mathrm{~Hz}$ and $248.6 \mathrm{~Hz}$ (curves superimposed in Figure 4). When the excitation force increases to $1 e^{-3} \mathrm{~N}$, a damping effect appears, and the two peaks are cut. If the excitation force is increased to $5 e^{-3} \mathrm{~N}$, the two peaks are even more damped, but the second peak is shifted to $248 \mathrm{~Hz}$. Finally, if the excitation force is again increased to $1 e^{-2} \mathrm{~N}$, the receptance for the first peak increases and the resonant frequency is slightly shifted of $0.1 \mathrm{~Hz}$. For the second peak, the resonant frequency continues to be shifted and is equal to $247.5 \mathrm{~Hz}$ and the receptance also increases.

\section{Optimisation Problem}

The objective is to optimise the topology of the damper in order to identify the geometry that gives the minimum level of vibrations at blade tips. Thus, the maximum of vibration amplitudes observed at blades tips over the frequency range $[100,400] \mathrm{Hz}$ is chosen as the objective function of the problem. The frequency range is chosen in order to cover all the possible variation of the resonance frequencies that may happen during the optimisation problem and to ensure a good initialisation of the MHBM. The limits of the design space are the limits of the full damper presented in Figure 3. The excitation amplitude is fixed to $1 e^{-3} \mathrm{~N}$ and is constant during the whole study. The loading range is directly related to the damper mass can take 
value between $0 \mathrm{~N}$ (damper of null mass) and $9.54 e^{-4} \mathrm{~N}$ (full damper).

\section{MOVING MORPHABLE COMPONENTS FRAMEWORK AND OPTIMIZATION PROCESS}

The objective of the following section is to present the methodology employed to perform topological optimization. The methodology is based on the KG-LSM [22], in other words the structure is represented implicitly by a Level-Set Function (LSF). The Kriging is used to surrogate this LSF and to update it to identify the best layout.

\section{Parametrization of the Problem}

Let's consider a bounded domain $\mathscr{D} \in \mathbb{R}^{2}$ which is the domain of all the admissible shapes. $\Omega$ denotes the region of the domain $\mathscr{D}$ occupied by the material. The LSF $\Phi$ that describes $\Omega$ is defined as:

$$
\left\{\begin{array}{l}
\Phi(\mathbf{v})>0, \mathbf{v} \in \Omega \\
\Phi(\mathbf{v})=0, \mathbf{v} \in \delta \Omega \\
\Phi(\mathbf{v})<0, \mathbf{v} \in D \backslash \Omega
\end{array}\right.
$$

where $\mathbf{v}=(x, y)^{\mathrm{T}} \in \mathscr{D}$ is a point of the domain, $\delta \Omega$ is the interface between the material and void. The 0-iso-line defines precisely the limit between material and void.

The idea of the Moving Morphable Components (MMC) framework, proposed by Guo et al. [21], is to decompose this global LSF into several local LSFs that describe an elementary beam component which can be moved and deformed in the design space. The idea relies on the fact that the optimal topologies can often be decomposed in an ensemble of interconnected beams. Hence, each local LSF $\Phi_{i}$ describes a domain $\Omega_{i}$ occupied by the component $i$. And the total domain $\Omega$ occupied is defined by $\Omega=\cup_{i=1}^{N} \Omega_{i}$.

And for $\mathscr{D}=\mathbb{R}^{2}$, each component $i$ is described by an explicit formulation of the LSF given by [21]:

$$
\begin{aligned}
\Phi_{i}(\mathbf{u})=- & {\left[\left(\frac{\cos \theta_{i}\left(x-x_{0 i}\right)+\sin \theta_{i}\left(y-y_{0 i}\right)}{l_{i} / 2}\right)^{m}+\right.} \\
& \left.\left(\frac{-\sin \theta_{i}\left(x-x_{0 i}\right)+\cos \theta_{i}\left(y-y_{0 i}\right)}{t_{i} / 2}\right)^{m}-1\right]
\end{aligned}
$$

where $\left(x_{0 i}, y_{0 i}\right)$ is the centre position of the component, $\theta_{i}$ is the inclination angle, $L$ the length and $t$ the thickness. $m$ is a relatively large even number taken equal to 6 here, as defined in [21]. Hence, each component is described by a set of five parameters, namely $\left(x_{0 i}, y_{0 i}, \theta_{i}, L, t\right)$, as illustrated in Figure 5 where the parametrization of a component is displayed. The corresponding

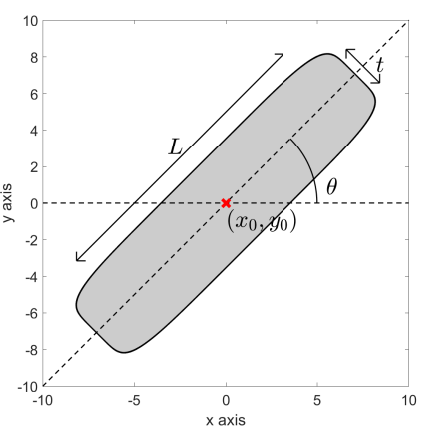

(a)

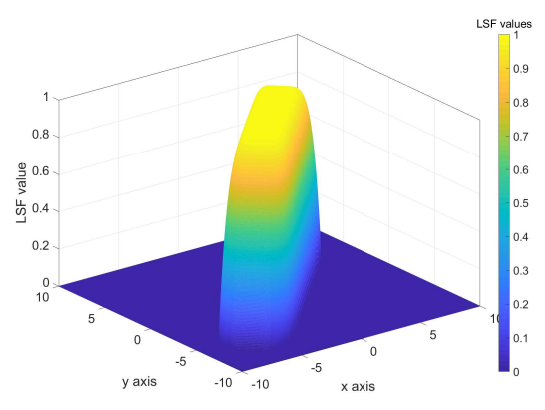

(b)
FIGURE 5: Parametrization of a component (a) and corresponding LSF (b)

local LSF is given in Figure $5 b$, where the negative values have been set to zero for display considerations. By changing these five parameters, each component can move and be distorted, and the assembly of the different components describes the general structure.

Once the structure is described by the LSF, this description must be mapped on the mechanical model and more precisely on the mesh. A classical method consists in having a fixed mesh on which the LSF is mapped with the use of an ersatz material: for the regions occupied by the material, the density of the material are used, but for the region composed of void, an ersatz material is used (i.e. with a density and a Young modulus largely lower than the material used). This allows to work on a fixed mesh and to always have a well-defined mechanical problem. This approach is largely used in the community [19-22,36]. However, in the computation of the eigenmodes of a structure, some can be localised in the ersatz material. To avoid this problem, the void elements are removed [37]. Hence, in the present study, the elements of the mesh that are not occupied by the material are removed. Moreover, the LSF is discretized in the centre of the elements [36], i.e. if the LSF is positive at the centre of an element of the mesh then this element is composed of material, but if the LSF is negative then the element is removed. This discretisation might seem a bit coarse, however the mesh can be refined (without additional numerical cost) for a more accurate description of the geometry of the damper. A second argument is that the topological optimization process is used to identify types of geometries that might be more efficient, but other design considerations need to be considered after and will define the final layout. To ensure a realisable component, the connectivity of the component is first checked with a $2 \mathrm{D}$ graph as proposed by Raponi et al. [22], and if check-board effect takes place then the thickness of the components is increased. An example of the global process is illustrated in Figure 6, where in Figure 6a 


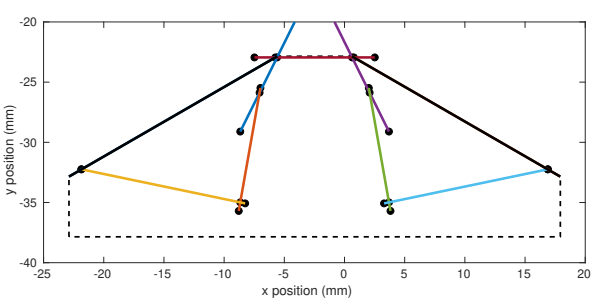

(a)

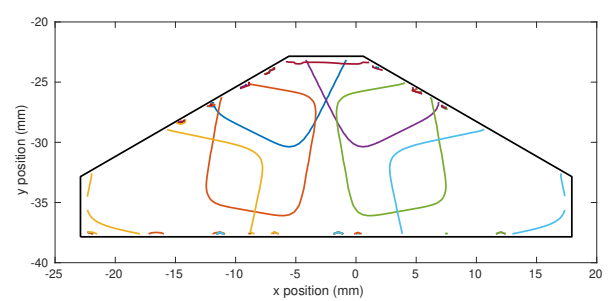

(b)

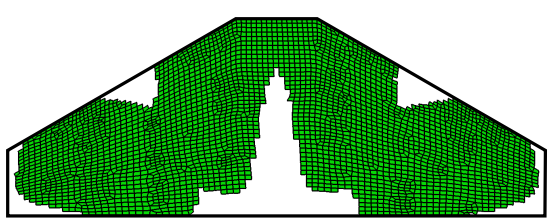

(c)

FIGURE 6: General geometry description: (a) 2D graph for the connectivity check - (b) 0-iso-contour of the different LSF - (c) corresponding mesh

a 2D graph is given. Each component is reduced to a segment and is represented by a colour. The limits of the design space are given in black. On Figure $6 \mathrm{~b}$, the 0 -iso-contour of each LSF is represented and finally the corresponding mesh is represented in Figure 6c.

\section{Optimization with Kriging and EGO Algorithm}

The optimization problem considered in this paper can be written under the form:

$$
\min _{\mathbf{x}} f_{\text {obj }}(\mathbf{x})
$$

where $f_{\text {obj }}$ is the objective function of the problem, namely the maximum of displacements at the blades tips. $\mathbf{x} \in \mathbb{R}^{n}$ is the vector of the design parameters that describes the geometry of the problem, which in this case corresponds to the number of LSF used multiplied by 5 , which are the describing parameters. The optimization problem is written without a constraint on the volume of the damper. This choice is driven by the fact that the most important goal in this study is to get a more efficient geometry, than a lighter one. Hence, no volume constraint is considered since it could lead to the missing of some efficient geometries.

The problem considered is strongly nonlinear, non-convex with many local minima, and the determination of the shape sensitivities of the objective function is not conceivable. Hence, an optimization strategy based on global optimization is considered. More precisely, the methodology is based on the use of a Kriging meta-model together with the Efficient Global Optimization (EGO) algorithm [23].

Kriging Surrogate Model Kriging is an approach to surrogate a function, that is often expensive to compute. In the present case, it surrogates the objective function $f_{\text {obj }}$. It is based on a set of $N$ inputs $\mathbf{X}=\left\{\mathbf{x}^{(1)}, \ldots, \mathbf{x}^{(N)}\right\}$ and their evaluations $\mathbf{y}=\left\{y^{(1)}, \ldots, y^{(N)}\right\}$. In the present case, each $y^{(k)}$ is obtained by computing the displacements FRF at blades tips with the MHBM, considering the damper geometry described by $\mathbf{X}^{(k)}$, over the frequency range $[100,400] \mathrm{Hz}$. The maximum over the frequency range and over the two blades is taken as the evaluation of the objective function. This set of inputs and outputs is called Design of Experiments (DoE). The set of input points is generated with a Latin Hypercube Sampling (LHS) and only the configurations that are connected are retained. Then, the Kriging methodology consists in approximating the function $f_{\text {obj }}$ by the function $\widehat{f}$ defined as [38]:

$$
\widehat{f}(\mathbf{x})=\sum_{j=1}^{q} g_{j}(\mathbf{x}) \beta_{j}+\mathscr{Z}(\mathbf{x})
$$

where $\left(g_{j}\right)_{j \in[1, q]}$ is a set of $q$ known real-values functions, $\left(\beta_{j}\right)_{j \in[1, q]}$ are the regression coefficients to be determined, and $\mathscr{Z}$ is a stationary zero-mean Gaussian process. Its covariance function is given by $C\left(\mathbf{x}, \mathbf{x}^{\prime}\right)=\sigma^{2} \mathscr{R}\left(\mathbf{x}, \mathbf{x}^{\prime}\right)$ where $\sigma^{2}$ is the unknown variance of the process and $\mathscr{R}\left(\mathbf{x}, \mathbf{x}^{\prime}\right)$ is the correlation function of the process. The functions $\left(g_{j}\right)_{j \in[1, q]}$ are chosen according to an a priori knowledge of the phenomenon, they are often low order polynomial functions. The function $\mathscr{R}$ is usually unknown but is often constructed from a family of kernel functions parametrized by a parameter $\theta$ to be optimized [38].

By assuming that $\mathscr{Z}$ is a Gaussian process, then the best unbiased predictor is linear. One can then define the regression matrix $\mathbf{G}$ of coefficients $G_{i j}=g_{j}\left(\mathbf{x}^{(i)}\right)$, and the correlation matrix $\mathbf{R}$ of coefficients $R_{i j}=C\left(\mathbf{x}^{(i)}, \mathbf{x}^{(j)}\right)$. The prediction $y_{0}$ of the Kriging meta-model at the point $\mathbf{x}_{0}$ is then given by:

$$
y_{0}=\widehat{f}\left(\mathbf{X}_{0}\right)=\mathbf{g}_{0}^{\mathrm{T}} \tilde{\beta}+\mathbf{r}_{0}^{\mathrm{T}} \mathbf{R}^{-1}(\mathbf{y}-\mathbf{G} \tilde{\beta})
$$

where $\mathbf{g}_{0}$ is the vector of $g_{j}\left(\mathbf{x}_{0}\right)$ and $\mathbf{r}_{0}$ is the vector of $C\left(\mathbf{x}_{0}, \mathbf{x}^{(i)}\right)$ and $\tilde{\beta}=\left(\mathbf{G}^{\mathrm{T}} \mathbf{R}^{-1} \mathbf{G}\right)^{-1} \mathbf{G}^{\mathrm{T}} \mathbf{R}^{-1} \mathbf{y}$. The mean square error is given 
by:

$$
\begin{aligned}
& s^{2}\left(\mathbf{x}_{0}\right)=\sigma^{2}\left(1-\mathbf{r}_{0}^{\mathrm{T}} \mathbf{R}^{-1} \mathbf{r}_{0}+\right. \\
& \left.\quad\left(\mathbf{g}_{0}-\mathbf{G}^{\mathrm{T}} \mathbf{R}^{-1} \mathbf{r}_{0}\right)^{\mathrm{T}}\left(\mathbf{G}^{\mathrm{T}} \mathbf{R}^{-1} \mathbf{G}\right)^{-1}\left(\mathbf{g}_{0}-\mathbf{G}^{\mathrm{T}} \mathbf{R}^{-1} \mathbf{r}_{0}\right)\right)
\end{aligned}
$$

EGO Algorithm The basic idea of the EGO algorithm consists in the creation of a Kriging surrogate model from an initial DoE which is updated at each iteration of the algorithm by adding a new point in the DoE [23]. The algorithm ends when a stop criterion is reached. The global work-flow of the method is given in Figure 7. Thus, the point added is based on the Expected Improvement (EI) criterion and is chosen in order to increase the knowledge in the area of the design space where the minimum of the objective function can be located, but also to explore areas where no knowledge is available. This is performed by using the meta-model created at the current step. The stop criterion is often based on a maximum number of iterations.

A modified EI proposed by Raponi et al. [22] is considered for this study. It is similar to the classical EI when the design corresponding to the points $\mathbf{x}$ is feasible (the connectivity between the components is ensured), and is penalised when the design is not feasible (i.e. when the components are not connected). Hence, at the point $\mathbf{x}$, the EI is defined as [22, 23,38]:

$$
\mathrm{EI}[\mathbf{x}]= \begin{cases}\left(y_{\min }-\widehat{f}(\mathbf{x})\right) \Phi\left(\frac{y_{\min }-\widehat{f}(\mathbf{x})}{\widehat{s}(\mathbf{x})}\right)+\widehat{s}(\mathbf{x}) \phi\left(\frac{y_{\min }-\widehat{f}(\mathbf{x})}{\widehat{s}(\mathbf{x})}\right), & \text { if feasible design and } \widehat{s}(\mathbf{x}) \neq 0, \\ 0, & \text { if feasible design and } \widehat{s}(\mathbf{x})=0, \\ -P * \gamma, & \text { if infeasible design, }\end{cases}
$$

where $y_{\min }$ is the current minimum, $\Phi$ is the Gaussian cumulative distribution function and $\phi$ is the Gaussian probability density function, $P$ is the minimum gap distance between the components and $\gamma \in \mathbb{R}^{+*}$ is the penalization factor (equal to 1000 here). The new point $\mathbf{x}_{\text {new }}$ is the solution that maximises the EI. This optimization problem is solved by using a genetic algorithm [39].

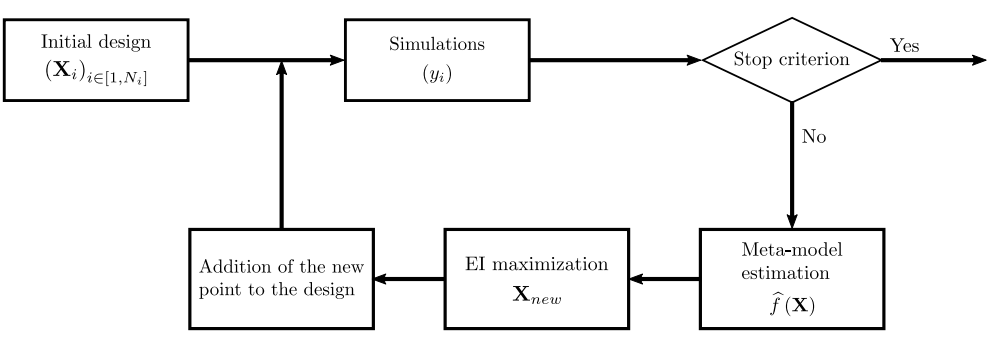

FIGURE 7: Algorithm EGO scheme

\section{Updating Limit Conditions}

For each point initially in the DoE and at each new point added during the process, a new damper geometry is described through the vector $\mathbf{x}$. Hence, the loading and the contact are also impacted and must be updated for each damper geometry. From the mass evolution of the damper, the centrifugal loading $C_{F}$ also evolves, and so the contact pressure and the contact stiffnesses are also impacted. The updating of the contact pressure is done by considering the new value of the centrifugal loading $C_{F}$, that depends on the damper mass, and also by considering the new contact area $A$, that is related to the damper geometry, in the Equation 1.

Moreover, the contact stiffnesses depends on the contact loading and so, they are also updated at each iteration by keeping the constant ratio [40]:

$$
\frac{k^{(1)}}{\sigma^{(1)}}=\frac{k^{(i)}}{\sigma^{(i)}}
$$

where $k^{(i)}$ is the contact stiffness and $\sigma^{(i)}$ the contact pressure at the iteration $i$. As a reminder, $k=k_{t}=k_{n}$.

\section{Summary Of The Global Process}

Finally, the global layout of the methodology can be described as follows. First the initialisation part can be decomposed as follows:

- Initialisation/step 1: a LHS is generated for the initial input points. Only the geometries that are connected are retained [22].

- Initialisation/step 2: the LSF corresponding to the design point is constructed and mapped on the initial damper mesh. The final damper mesh is created by eliminating the void elements.

- Initialisation/step 3: for each damper geometry, the nonlinear dynamic response of both the platform and the damper is determined with the MHBM where the contact pressure and contact stiffness have been updated. The excitation amplitude is constant and equal to $1 e^{-3} \mathrm{~N}$. The maximum of displacements at the blades tips over the frequency range $[200,400] \mathrm{Hz}$ is retained as the evaluation of the objective function.

and the general optimisation loop can be decomposed as follows:

- Loop/step 1: from the DoE, a surrogate model is generated.

- Loop/step 2: from the surrogate model, the EI is maximized and the new point added to the design.

- Loop/step 3: for this point (i.e. new geometry), the objective function is determined (steps 2 and 3 of the initialisation).

The loop is repeated until the stop criterion is reached. Concerning some practical aspects, in the present study, the global work- 
flow is piloted by Matlab. The package DiceKriging [38] on $\mathrm{R}$ is used for the creation of the different Kriging meta-models. A kernel Matérn 5/2 is used and the regression function is polynomial of order 0 .

\section{APPLICATION}

The methodology presented previously is then applied to optimize the topology of the damper. Seven components are used to describe the geometry of the damper, as shown in Figure 6, which would represent 35 optimization parameters. In order to reduce this number of parameters, some assumptions are done. First, a component is assumed to be horizontal at the top of the design space and aims to seal the platforms. This constraint comes from the fact that the primary function of a UPD is to seal the platforms to ensure the aerodynamic efficiency. Second, the damper

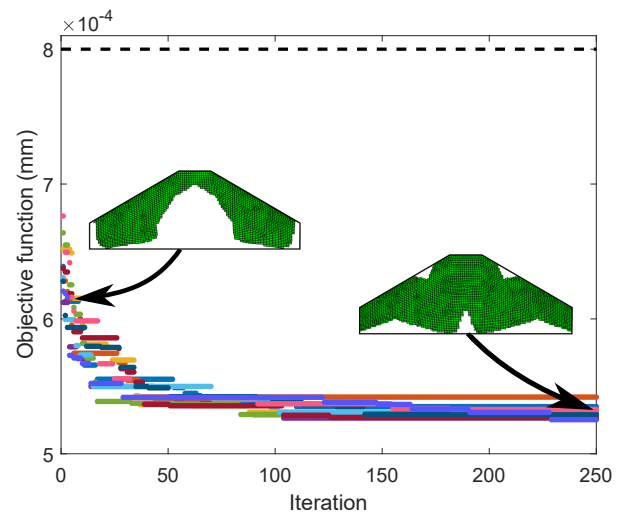

(a) One color corresponds to one DoE - (- - -) full damper (reference) - Initial and final geometries for one DoE

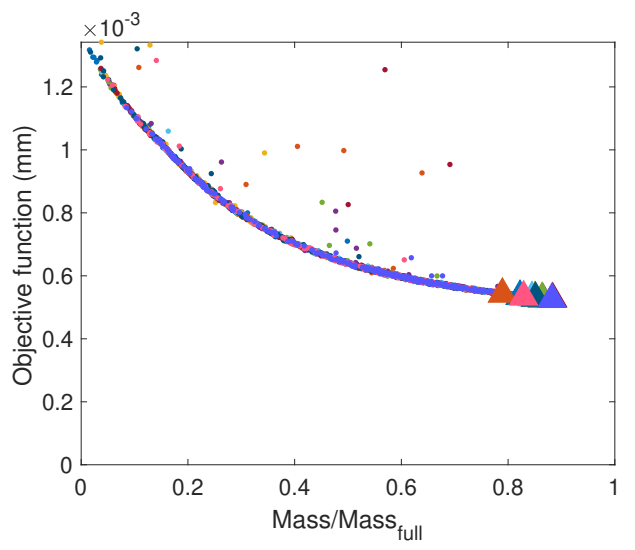

(b) One color corresponds to one $\operatorname{DoE}-(\triangle)$ final solution

FIGURE 8: (a) Evolution of the objective function (i.e. max of displacements at blades tips) - (b) Objective function versus the normalized mass of the damper for all the different configurations tested during the algorithm is supposed to be symmetric. And finally, one component has its centre position imposed to always have a contact between the damper and the platforms. This restriction ensures just a feasible design. Thanks to these restrictions, the final optimization problem is composed of 14 parameters. As a reminder, the excitation amplitude remains constant and equal to $1 e^{-3} \mathrm{~N}$.

\section{Results}

To ensure the validity and the robustness of the method, ten initial DoE are created, and ten simulations are launched. In each case, an initial LHS of $10^{5}$ points is generated, and only the feasible geometries are kept (i.e. no connectivity issue). Depending on the case, it represents $[65,51,65,62,62,56,82,61,62,81]$ initial learning points for the ten cases. In order to get reasonable computation time, a maximum of 250 iterations is chosen. Hence, the evolution of the current minimum at each iteration for the ten cases is given in Figure 8a where each colour corresponds to one case. At the first iteration, the minimum is the one observed in the initial DoE, depending on the case it is included between 6 and $6.8 e^{-4} \mathrm{~mm}$. Then, the evolution of the minima is quite similar for all cases. First the decrease of the minimum is fast. Indeed, in about 40 iterations, all the minima are below $5.5 e^{-4} \mathrm{~mm}$. After 40 iterations, the decrease becomes slower and stabilizes around 150 iterations. For all cases, the algorithm converges to a solution from 5.3 to $5.4 e^{-4} \mathrm{~mm}$. It is worth reminding that for the full damper the maximum level of amplitudes is equal to $8 e^{-4} \mathrm{~mm}$. Hence, the algorithm identifies here ten solutions that are $30 \%$ more efficient than the original one. As explained previously, no constraint on the volume is considered in this application. It may sound peculiar since the first objective of topological optimization is to reduce the weight of the structure. In this case, the most important objective is to achieve better design. Considering a constraint on the weight could lead to miss some interesting layouts. However, it is still an interesting argument to consider a solution instead of another one. Hence, in Figure $8 \mathrm{~b}$ represents the maximum of displacements versus the normalized mass for all the configurations tested with the ten cases. It appears that almost all the configurations tested are located on a front. It shows also that by minimizing the maximum of displacements, the mass increases, i.e. the two functions are antagonist. The last configuration identified in each case, i.e. the current minimum at the iteration 250, is represented on the graph with a triangle. They are all located in the same part of the graph and so have similar masses.

The ten final configurations are given in Figure 9. The green part corresponds to the mesh associated to each geometry, and the borders of the design space are reminded. It can be seen that the geometries are all different. Indeed, some have holes inside the structure (see in Figures 9b, 9d, 9j), some cut the contact surface into two parts (see in Figures 9a, 9e, 9f, 9i). However, some geometries look similar and some groups can be done. For exam- 


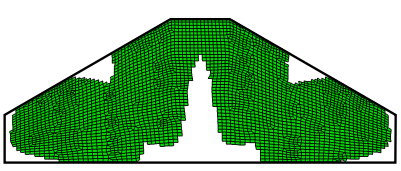

(a) Damper geometry from DoE1

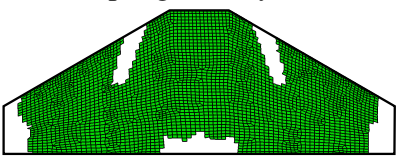

(e) Damper geometry from DoE5

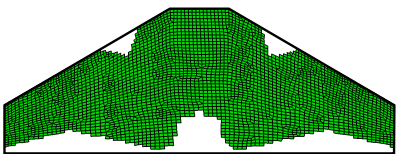

(i) Damper geometry from DoE9

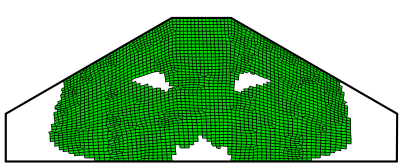

(b) Damper geometry from DoE2

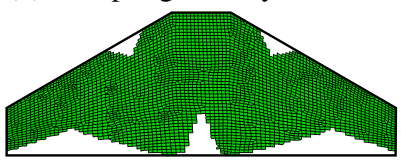

(f) Damper geometry from DoE6

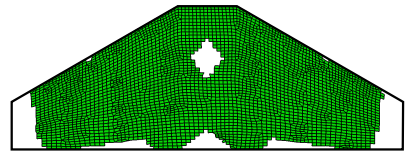

(j) Damper geometry from DoE10

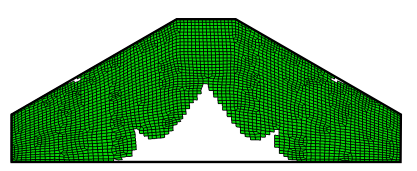

(c) Damper geometry from DoE3

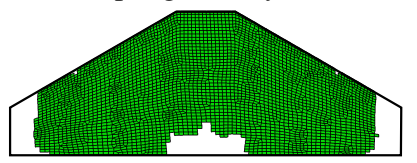

(g) Damper geometry from DoE7

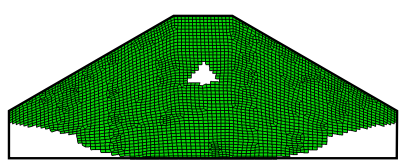

(d) Damper geometry from DoE4

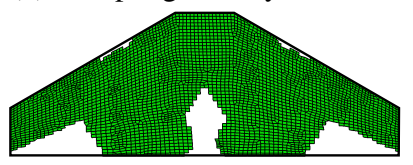

(h) Damper geometry from DoE8

FIGURE 9: Damper geometries obtained from the different DoE

ple, geometries of Figures 9a, 9f and 9i have very similar layouts. It appears that the FRF of each configuration are almost identical so only one of them in represented in the following. The FRF is given in Figure 10a for the tip point on the left blade and in Figure $10 \mathrm{~b}$ for the right blade. The FRF corresponding to the solutions of the optimization are in dark blue, and the FRF of the full damper is represented with the black dotted line. They are given only for the horizontal displacement. In the initial case, the first peak was located at $246.6 \mathrm{~Hz}$ and the second at $248.8 \mathrm{~Hz}$. The FRFs of the optimized geometries have a first peak shifted on the right (new peak at $247.2 \mathrm{~Hz}$ ), whereas the second one is shifted on the left (new peak at $248 \mathrm{~Hz}$ ). The same phenomenon appears on the second node: the first peak is shifted from $246.7 \mathrm{~Hz}$ to $247.5 \mathrm{~Hz}$ and the second from $248.5 \mathrm{~Hz}$ to $248 \mathrm{~Hz}$. Moreover, in the initial case, the FRF of the two dof were similar: the first peak has the largest amplitude and the second the smaller. Whereas after optimization, for the second dof, the second peak is the one with the largest amplitude.

In order to observe the efficiency of lighter dampers, the FRF of other geometries tested during the optimization process are considered. Geometries with a mass equal to $50 \%, 30 \%$ and $10 \%$ of the total mass $M_{\text {full }}$ are considered. One of each case is represented in Figure 11. As previously, for one mass, all the FRF are extremely similar, hence only one FRF is retained in each case for the comparison. The different FRF are represented in Figure 10. The black dashed line corresponds to the initial case (full damper). For the first dof, a decrease of the mass tends to increase the amplitude of the first peak: $1.1 e^{-3} \mathrm{~mm}$ of amplitude if $M=0.1 M_{\text {full }}$ and $0.54 e^{-3} \mathrm{~mm}$ if $M=0.9 M_{\text {full }}$. For the second peak, a lighter damper tends to decrease slightly the amplitudes. Moreover, the all FRF tends to be shifted on the left when the damper is lighter. Considering the second dof (see in
Figure $10 \mathrm{~b}$ ), when the damper has a mass $M$ equal to $0.1 M_{\text {full }}$, the two peaks have similar amplitudes. The increase of the mass tends to reduce the amplitude of the first peak, whereas the amplitude of the second peak remains sensibly constant. As for the other dof, the FRF is shifted on the right when the mass increases.

\section{CONCLUSION}

In the present study, TO is applied to optimise nonlinear vibrations in the presence of a friction interface, which is completely new for the community from authors knowledge. A simple 2D model composed of two blades and a UPD, where the topology of the damper is optimised, is used as a test case. The TO method is based on the MMC framework to parametrize the LSF that describes the geometry of the system. A Kriging method together with the EGO algorithm is used as optimisation process. The study was aimed to investigate the capacity of the MMC framework coupled with a global optimisation algorithm to perform TO for nonlinear vibrations in a presence of a friction interface. Ten simulations based on different DoE are launched to verify the validity and the robustness of the approach. The results show a large variety of UPD geometries, but they all have very similar effectiveness and almost identical nonlinear dynamic response. The optimised geometries lead to a reduction of about $30 \%$ of the nonlinear vibration levels of the structure compared to the case with the full damper. The results obtained in the study tends to show the promising aspect of using the MMC framework coupled with EGO algorithm for TO of UPD. Nevertheless, a deeper investigation must be conducted to take into consideration different mechanical constraints, as the stress, to obtain more realistic layouts that can tolerate the operating conditions. 


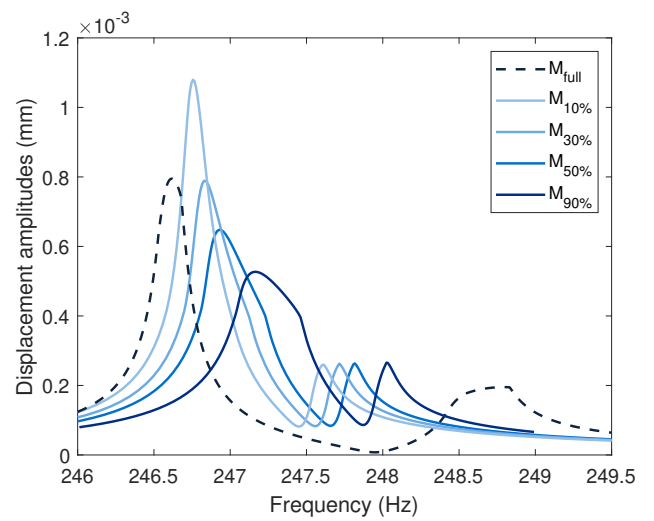

(a) Left blade

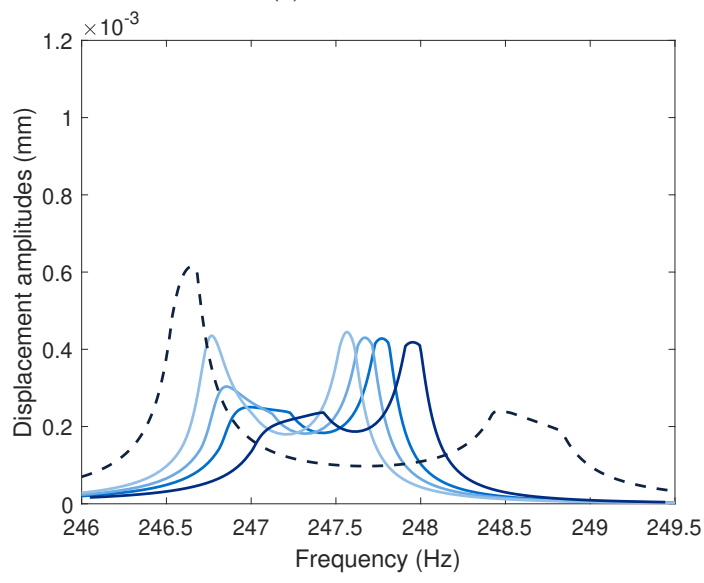

(b) Right blade

FIGURE 10: Displacements amplitudes at blades tip nodes for different masses of damper: Full damper, $M=90 \% M_{\text {full }}, M=$ $50 \% M_{\text {full }}, M=30 \% M_{\text {full }}, M=10 \% M_{\text {full }}$

\section{ACKNOWLEDGMENT}

The authors thank Rolls-Royce plc and the EPSRC for the support under the Prosperity Partnership Grant "Cornerstone: Mechanical Engineering Science to Enable Aero Propulsion Futures", Grant Ref: EP/R004951/1.

\section{REFERENCES}

[1] Krack, M., Salles, L., and Thouverez, F., 2017. "Vibration prediction of bladed disks coupled by friction joints". Archives of Computational Methods in Engineering, 24(3), pp. 589-636.

[2] Petrov, E., and Ewins, D., 2004. "State-of-the-art dynamic analysis for non-linear gas turbine structures". Proceedings of the Institution of Mechanical Engineers, Part G: Journal of Aerospace Engineering, 218(3), pp. 199-211.

[3] Cowles, B., 1996. "High cycle fatigue in aircraft gas tur-

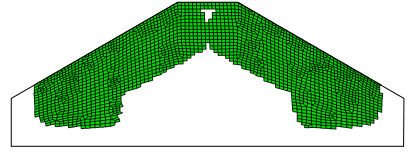

(a) $M=0.5 M_{\text {full }}$

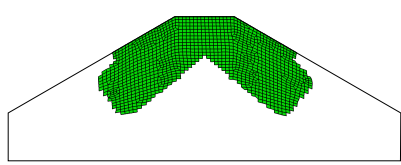

(b) $M=0.3 M_{\text {full }}$

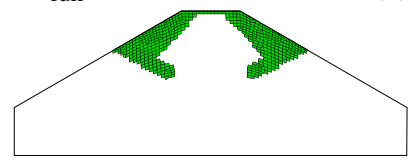

(c) $M=0.1 M_{\text {full }}$

FIGURE 11: Lighter geometries of damper observed during the optimization

bines-an industry perspective". International Journal of Fracture, 80(2-3), pp. 147-163.

[4] Gaul, L., and Nitsche, R., 2001. "The role of friction in mechanical joints". Applied Mechanics Reviews, 54(2), pp. 93-106.

[5] Feeny, B., Guran, A., Hinrichs, N., and Popp, K., 1998. “A historical review on dry friction and stick-slip phenomena".

[6] Griffin, J., 1990. "A review of friction damping of turbine blade vibration". International Journal of Turbo and Jet Engines, 7(3-4), pp. 297-308.

[7] Szwedowicz, J., Gibert, C., Sommer, T., and Kellerer, R., 2008. "Numerical and experimental damping assessment of a thin-walled friction damper in the rotating setup with high pressure turbine blades". Journal of Engineering for Gas Turbines and Power, 130(1), p. 012502.

[8] Sanliturk, K., Ewins, D., and Stanbridge, A., 1998. "Underplatform dampers for turbine blades: theoretical modeling, analysis, and comparison with experimental data". J. Eng. Gas Turbines Power, 123(4), pp. 919-929.

[9] Sanliturk, K., Ewins, D., Elliott, R., and Green, J., 1999. "Friction damper optimization: simulation of rainbow tests". J. Eng. Gas Turbines Power, 123(4), pp. 930-939.

[10] Petrov, E., and Ewins, D., 2007. "Advanced modeling of underplatform friction dampers for analysis of bladed disk vibration". Journal of Turbomachinery, 129(1), pp. 143150.

[11] Panning, L., Sextro, W., and Popp, K., 2000. "Optimization of interblade friction damper design". In ASME Turbo Expo 2000: Power for Land, Sea, and Air.

[12] Pesaresi, L., Salles, L., Jones, A., Green, J., and Schwingshackl, C., 2017. "Modelling the nonlinear behaviour of an underplatform damper test rig for turbine applications". Mechanical Systems and Signal Processing, 85, pp. 662679.

[13] Csaba, G., 1999. "Modelling of a microslip friction damper subjected to translation and rotation". In ASME 1999 international gas turbine and aeroengine congress and exhibition. 
[14] Zucca, S., Botto, D., Gola, K., and Muzio, M., 2008. "Range of variability in the dynamics of semi-cylindrical friction dampers for turbine blades". In ASME Turbo Expo 2008: Power for Land, Sea, and Air, pp. 519-529.

[15] Rozvany, G., and Zhou, M., 1993. "Layout and generalized shape optimization by iterative coc methods". In Optimization of large structural systems. Springer, pp. 103-120.

[16] Eschenauer, H., and Olhoff, N., 2001. "Topology optimization of continuum structures: a review". Applied Mechanics Reviews, 54(4), pp. 331-390.

[17] Bendsøe, M., 1989. "Optimal shape design as a material distribution problem”. Structural optimization, 1(4), pp. 193-202.

[18] Philip, M. B., and Kikuchi, N., 1988. "Generating optimal topologies in structural design using a homogenization method". Computer methods in applied mechanics and engineering, 71(2), pp. 197-224.

[19] van Dijk, N., Maute, K., Langelaar, M., and Keulen, F. V., 2013. "Level-set methods for structural topology optimization: a review". Structural and Multidisciplinary Optimization, 48(3), pp. 437-472.

[20] Allaire, G., Jouve, F., and Toader, A.-M., 2004. "Structural optimization using sensitivity analysis and a levelset method". Journal of computational physics, 194(1), pp. 363-393.

[21] Guo, X., Zhang, W., and Zhong, W., 2014. "Doing topology optimization explicitly and geometrically_a new moving morphable components based framework". Journal of Applied Mechanics, 81(8), p. 081009.

[22] Raponi, E., Bujny, M., Olhofer, M., Aulig, N., Boria, S., and Duddeck, F., 2019. "Kriging-assisted topology optimization of crash structures". Computer Methods in Applied Mechanics and Engineering, 348, pp. 730-752.

[23] Jones, D. R., Schonlau, M., and Welch, W. J., 1998. "Efficient global optimization of expensive black-box functions". Journal of Global optimization, 13(4), pp. 455492.

[24] Sun, J., Tian, Q., Hu, H., and Pedersen, N. L., 2019. "Topology optimization for eigenfrequencies of a rotating thin plate via moving morphable components". Journal of Sound and Vibration, 448, pp. 83-107.

[25] Bendsøe, M. P., and Díaz, A. R., 1994. "Optimization of material properties for improved frequency response". Structural optimization, 7(1-2), pp. 138-140.

[26] Díaaz, A. R., and Kikuchi, N., 1992. "Solutions to shape and topology eigenvalue optimization problems using a homogenization method". International Journal for Numerical Methods in Engineering, 35(7), pp. 1487-1502.

[27] Allaire, G., and Jouve, F., 2005. "A level-set method for vibration and multiple loads structural optimization". Computer methods in applied mechanics and engineering, 194(30-33), pp. 3269-3290.
[28] Dou, S., and Jensen, J. S., 2015. "Optimization of nonlinear structural resonance using the incremental harmonic balance method". Journal of Sound and Vibration, 334, pp. 239-254.

[29] Dou, S., Strachan, B. S., Shaw, S. W., and Jensen, J. S., 2015. "Structural optimization for nonlinear dynamic response". Philosophical Transactions of the Royal Society A: Mathematical, Physical and Engineering Sciences, 373(2051), p. 20140408.

[30] Myśliński, A., 2012. "Topology optimization of quasistatic contact problems". International Journal of Applied Mathematics and Computer Science, 22(2), pp. 269-280.

[31] Strömberg, N., and Klarbring, A., 2010. "Topology optimization of structures in unilateral contact". Structural and Multidisciplinary Optimization, 41(1), pp. 57-64.

[32] Maury, A., Allaire, G., and Jouve, F., 2017. "Shape optimisation with the level set method for contact problems in linearised elasticity". The SMAI journal of computational mathematics, 3, pp. 249-292.

[33] Petrov, E., 2008. "Explicit finite element models of friction dampers in forced response analysis of bladed disks". Journal of engineering for gas turbines and power, 130(2), p. 022502.

[34] Petrov, E., 2011. "A high-accuracy model reduction for analysis of nonlinear vibrations in structures with contact interfaces". Journal of Engineering for Gas Turbines and Power, 133(10), p. 102503.

[35] Cameron, R., and Martin, W., 1947. "The orthogonal development of non-linear functionals in series of fourierhermite functionals". Annals of Mathematics, pp. 385-392.

[36] Challis, V., 2010. "A discrete level-set topology optimization code written in matlab". Structural and multidisciplinary optimization, 41(3), pp. 453-464.

[37] Li, Z., Shi, T., and Xia, Q., 2017. "Eliminate localized eigenmodes in level set based topology optimization for the maximization of the first eigenfrequency of vibration". Advances in Engineering Software, 107, pp. 59-70.

[38] Roustant, O., Ginsbourger, D., and Deville, Y., 2012. "Dicekriging, diceoptim: Two $\mathrm{r}$ packages for the analysis of computer experiments by kriging-based metamodeling and optimization".

[39] Goldberg, D., and Holland, J., 1988. "Genetic algorithms and machine learning”. Machine learning, 3(2), pp. 95-99.

[40] Sextro, W., 2007. Dynamical contact problems with friction. Springer. 